\title{
Numerical characteristics of a centrifugal compressor with a low flow coefficient
}

\author{
Aleksey Yablokov ${ }^{1, *}$, Ivan Yanin ${ }^{1}$, Nikolay Sadovskyi ${ }^{1}$, Yuri Kozhukhov ${ }^{1}$ and Minh Hai Nguyen ${ }^{2}$ \\ ${ }^{1}$ Peter the Great St. Petersburg Polytechnic University, St. Petersburg, Russian Federation \\ ${ }^{2}$ Petrovietnam exploration production corporation Integrated technical center
}

\begin{abstract}
The study presents the simulation results of the viscid gas flow in low flow coefficient centrifugal compressor stages. The problem is solved in a stationary formulation using the Ansys CFX software package. The numerical simulation is carried out on three ultrahigh-pressure model stages; two stages have blades of the classical type impeller and one stage is of the bodily type. The value of the conditional flow coefficient is 0.0063 to 0.015 . As part of the study, block-structured design meshes are used for all gas channel elements, with their total number being equaled as 13-15 million. During the calculations a numerical characteristic was validated with the results of tests carried out at the Department of Compressor, Vacuum and Refrigeration Engineering of Peter the Great St. Petersburg Polytechnic University. With an increase of inlet pressure as a result of a numerical study, it was found that for a given mathematical model the disk friction and leakage coefficient $(1+\beta \mathrm{fr}+\beta \mathrm{lk})$ is overestimated. The analysis of flow in labyrinth seals has shown an increase of total temperature near the discs by $30-50{ }^{\circ} \mathrm{C}$, nevertheless this fact did not influence gas parameters in the behind-the-rotor section. The calculation data obtained with finer design mesh (the first near-wall cell was $0.001 \mathrm{~mm}$ ) is identical to those obtained with the first near-wall cell $0.01 \mathrm{~mm}$ mesh.
\end{abstract}

\section{Introduction}

Low flow coefficient centrifugal compressors are used to obtain high and ultrahigh gas pressures, which is necessary for modern gas transport systems. So the efficiency increasing of these compressors is an urgent task, especially for the oil and gas industry. The main reason for the efficiency decrease in the low flow stages compressors is the low volumetric flow. And as a result of this are narrow flow sections, small hydraulic diameters, and low Reynolds numbers. This fact was confirmed in works [1, 2, 3], related to the thermo-gasdynamic principles ultrahigh-pressure centrifugal compressors design and experimental studies. The unsteady nature of the gas flow in the stage of a centrifugal compressor also plays an important role. In the work [4] showed that the amplitude of the pressure change in the peripheral sections of the impeller reaches $4 \mathrm{MPa}$. According to the results of calculating the unsteady flow in the work [1], a conclusion was made about the undesirability of using traditional design blade diffusers. Low flow rates with small channel sizes require increased manufacturing accuracy and minimal surface roughness, which leads to additional cost increases.

In the work [5], the flow sections losses of a centrifugal compressor are conventionally divided into 5 groups:

-Channel friction losses;
-Vortex loss;

-Secondary losses;

-Losses on internal leakage;

-Loss on disk friction of the outer surfaces of the impellers.

In this article, the authors pay special attention to the 4th and 5th groups, since these losses make up a significant part of all losses in low flow coefficient centrifugal compressor stages.

\section{Methods}

As an object of study, the authors of the article selected model stages of a ultra-high pressure centrifugal compressor designed, manufactured and tested on a closed-loop stand at the department of the compressor, vacuum and refrigeration Engineering of Peter the Great St. Petersburg Polytechnic University. For model stages, the values of the theoretical flow coefficient lie in the range $0.0063-0.015$ and are calculated by the formula:

$$
\Phi=\frac{4 \bar{m}}{\rho_{0}^{*} \pi D_{2}^{2} U_{2}},
$$

where $\bar{m}$ - mass flow, $\mathrm{kg} / \mathrm{s} ; \rho_{0}^{*}-$ inlet gas density, $\mathrm{kg} / \mathrm{m}^{3} ; D_{2}-$ outlet diameter of the impeller, $\mathrm{m} ; U_{2}-$ circumferential velocity on the diameter $D_{2} \mathrm{~m} / \mathrm{s}$;

\footnotetext{
* Corresponding author: yablokovaleksey@mail.ru
} 
Table 1. The number of design mesh elements for numerical models of centrifugal compressor stages.

\begin{tabular}{|c|c|c|c|c|}
\hline Name title of the stage & $\begin{array}{c}\text { Optimal flow } \\
\text { factor } \boldsymbol{\Phi}_{\text {opt }}\end{array}$ & $\begin{array}{c}\text { Theoretical } \\
\text { head factor } \boldsymbol{\Psi}_{\mathbf{T}}\end{array}$ & $\begin{array}{c}\text { Averaged } \\
\text { bore, } \mathbf{m}\end{array}$ & $\begin{array}{c}\text { The number of rotor } \\
\text { blades Zimp }\end{array}$ \\
\hline Model stage SVD-1 & 0.083 & 0.48 & 0.497 & 12 \\
\hline Model stage SVD-1 & 0.08 & 0.48 & 12 \\
\hline
\end{tabular}

Table 1 presents the main parameters of the model stages SVD-1 and SVD-2 with cylindrical blades.

The model stage of the SVD-6 series has an impeller with body-shaped blades designed with $\Phi_{\text {opt }}=0.015$; $\Psi_{\mathrm{T}}=0,545 ; \bar{D}_{h u b}=0.4$. The number of impeller blades $\mathrm{Z}_{\text {imp }}=8$. The impeller SVD-6 was designed so that the equivalent opening angle of the channels does not exceed 10 degrees. The channels, as well as the outer surfaces of the SVD - 6 disks, have a roughness $\mathrm{Ra}=5$ 2.5. The return channel middle line blades are made along an arc of a circle. The blade angle at the exit of the return channel is $90^{\circ}$, and at the entrance is $25^{\circ}$. The number of blades is 16 . A vaneless diffuser with $\mathrm{D}_{4} / \mathrm{D}_{2}$ $=1.55$ is installed in the stage. Figure 1 shows the schematic diagram of the SVD-6 intermediate stage rotor.

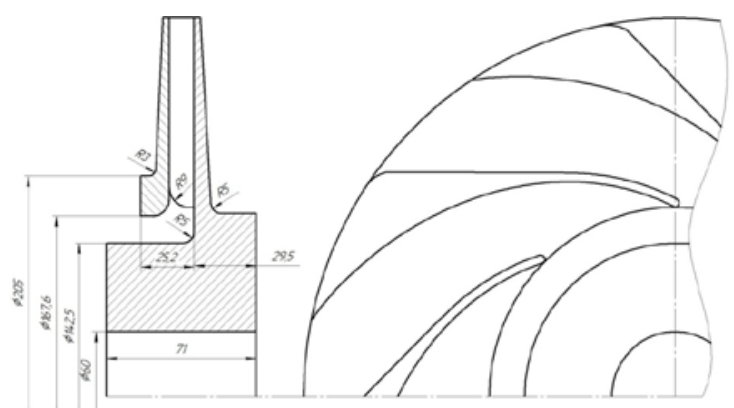

Fig. 1. A schematic diagram of the SVD-6 intermediate stage rotor.

The numerical simulation of the viscid gas flow in the model stages SVD channels was carried out in the
Ansys CFX software package in a stationary formulation with a subsonic gas flow. The flowing gas channels centrifugal compressor stage model was the volume enclosed between the bounding surfaces of the real stage. This volume was completely filled with a blockstructured grid. The number of elements of the computational grid of model stages, rounded to thousands, is given in Table 2. Various boundary conditions were set on the model surfaces. The numerical study model consists of the elements shown in Figure 2.

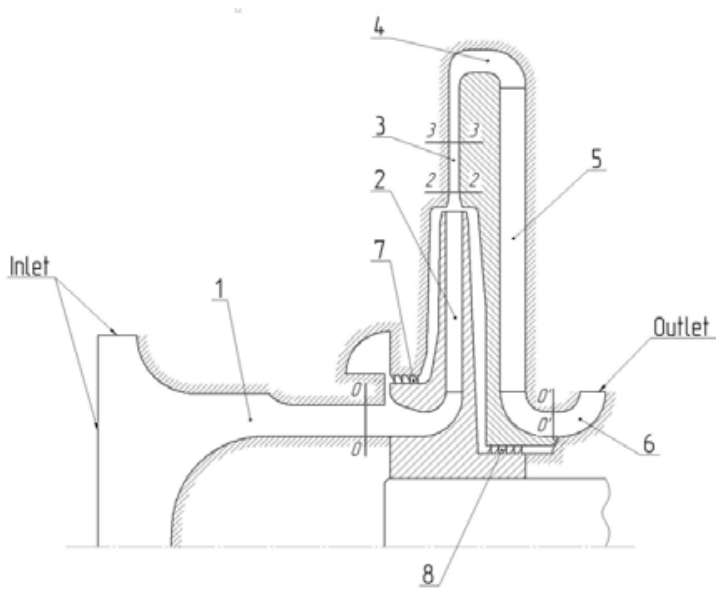

Fig. 2. A gas channel schematic diagram of the stage in meridional plane: 1 - inlet guide; 2 - rotor; 3 - vaneless diffuser; 4 - crossover; 5 - return channel; 6 - output guide; 7 - labyrinth seals along the covering disc; 8 - labyrinth seals along the main disc.

Table 2. The number of design mesh elements for numerical models of centrifugal compressor stages.

\begin{tabular}{|c|c|c|c|}
\hline № & Name title of the stage & Total number of elements, mln & Number of impeller elements, mln \\
\hline 1 & Model stage SVD-1 & 15700000 & 3000000 \\
\hline 2 & Model stage SVD-2 & 14000000 & 2800000 \\
\hline 3 & Model stage SVD-6 & 13000000 & 2500000 \\
\hline
\end{tabular}

Table 3. Input parameters for designing of model stage.

\begin{tabular}{|c|c|c|c|}
\hline № & Name title of the stage & Inlet pressure, MPa & Gas type \\
\hline \multirow{3}{*}{1} & \multirow{3}{*}{ Model stage SVD-1 } & 0.1 & Air ideal gas \\
\cline { 3 - 4 } & & 0.41 & Nitrogen N2 \\
\cline { 3 - 4 } & \multirow{3}{*}{2} & 1.0 & Nitrogen N2 \\
\cline { 3 - 4 } & \multirow{3}{*}{3} & 0.1 & Air ideal gas \\
\cline { 3 - 4 } & Model stage SVD-2 & 0.41 & Nitrogen N2 \\
\cline { 3 - 4 } & \multirow{3}{*}{ Model stage SVD-6 } & 1.0 & Nitrogen N2 \\
\cline { 3 - 4 } & & 0.1 & Air ideal gas \\
\cline { 3 - 4 } & & 0.41 & Nitrogen N2 \\
\cline { 3 - 4 } & & 1.0 & Nitrogen N2 \\
\cline { 3 - 4 } & & 2.0 & Nitrogen N2 \\
\hline
\end{tabular}


Table 3 shows the boundary condition parameters at the entrance to the computational domain. Mass flow was set at the exit from the calculation area. It was calculated by the input parameters through the conditional flow coefficient $\Phi$.

The SST turbulence model was used for all low flow stage models. The value of the near-wall function $y+$ is less than 3. The calculated block-structured grids were designed according to the recommendations of the authors of the works [6-18]. The results of the work were obtained using computational resources of Peter the Great Saint-Petersburg Polytechnic University Supercomputing Center (www.spbstu.ru).

\section{Results and Discussion}

The methodology of the Department KViHT was used to process the results of a numerical study and the following main parameters were calculated:

1. The conditional flow coefficient:

$$
\Phi=\frac{\overline{4 m}}{\rho_{0}^{*} \pi D_{2}^{2} U_{2}}
$$

The polytrophic pressure coefficient:

$$
\psi_{p}=\frac{h_{p}}{U_{2}^{2}}
$$

where $h_{\mathrm{p}}$ - polytropic head, J/kg;

2. The coefficient of polytropic head with the difference of the kinetic energies of the gas:

$$
\psi_{p}^{*}=\frac{h_{p}^{*}}{U_{2}^{2}}
$$

3. The coefficient of internal head:

$$
\begin{gathered}
\psi_{i}=\frac{h_{i}}{U_{2}^{2}} \\
h_{i}=\frac{N_{i}}{U_{2}^{2}}
\end{gathered}
$$

where $h_{i}$ - internal head, $\mathrm{J} / \mathrm{kg} ; \mathrm{N}_{\mathrm{i}}$ - engine power transmitted to the gas by the impeller of the stage, $\mathrm{N} / \mathrm{m}^{2}$.

For the case of insignificant influence of heat transfer, the calculation is carried out according to the formula:

$$
h_{i}=i_{0^{\prime}}^{*}-i_{0}^{*}
$$

4. Polytropic efficiency by static parameters:

$$
\eta_{p}=\frac{h_{p}}{h_{i}-h_{d}}
$$

5. Polytropic efficiency by complete parameters:

$$
\eta_{p}^{*}=\frac{h_{p}^{*}}{h_{i}}
$$

6. Power friction with the friction torque

$$
N_{f r}=\omega \cdot M_{f r}=k_{f r} \rho U_{2}^{3} D_{2}^{2}\left(1-\frac{D_{1}^{2}}{D_{2}^{2}}\right) \cdot 10^{-6}
$$

where $k_{f r}=\pi \lambda_{1} \cdot 10^{2} \quad-$ coefficient dependent on Reynolds number $\operatorname{Re}_{u}=\frac{U_{2} D_{2}}{2 v_{2}}$, surface roughness of the disk and relative to the lateral clearance $\frac{b_{2}}{D_{2}}$ between the disc and the housing, determined according to Tsumbush.

Figures 3, 4 show the characteristics of the model stage SVD - 1 according to the results of a numerical study in comparison with the experimental results. The calculated input pressure is $0.1 \mathrm{MPa}$ of air ideal gas. The characteristics are built in section 2-2.

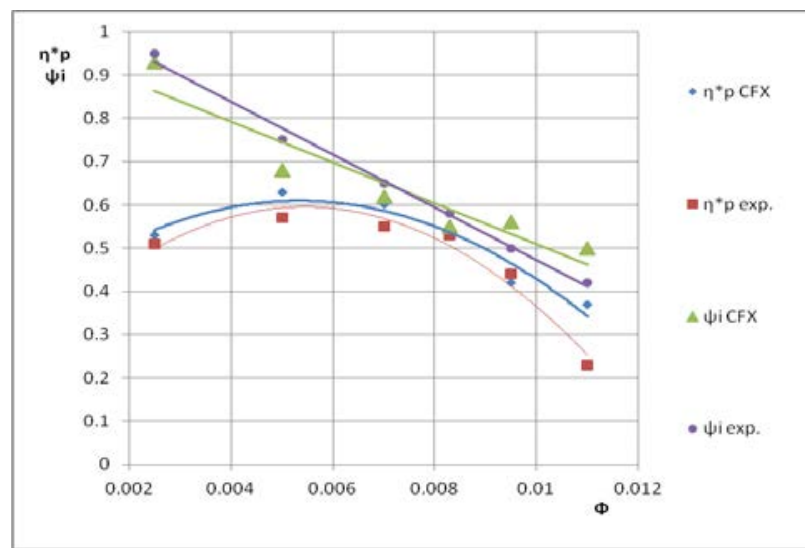

Fig. 3. Estimated and experimental characteristics of SVD-1 stage at $\mathrm{Re}_{\mathrm{u}}=2.5 \cdot 10^{6}$ in section $2-2$. Inlet pressure $\mathrm{P}=0.1 \mathrm{MPa}$, actuating medium - ideal gas.

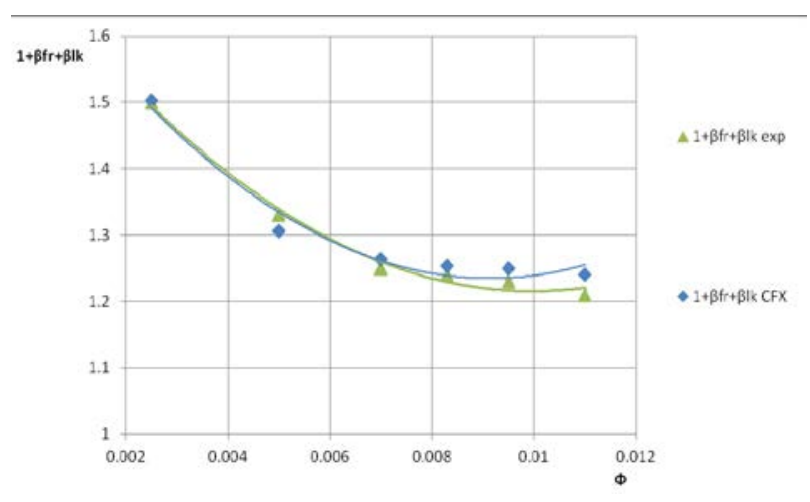

Fig. 4. Estimated and experimental characteristics of cumulative leak and disk friction loss factor $\left(1+\beta_{\mathrm{fr}}+\beta_{\mathrm{lk}}\right)$. Inlet pressure $\mathrm{P}=0.1 \mathrm{MPa}$, actuating medium - ideal gas.

Figures 5 show the calculated and experimental characteristics of the SVD-1 stage with an inlet pressure 
of $0.4 \mathrm{MPa}$. A real nitrogen gas model was used to simulate a viscid gas flow.

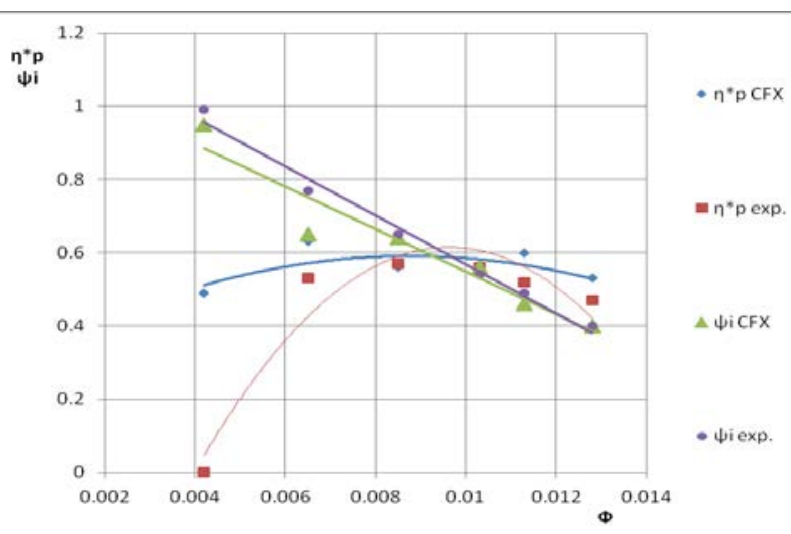

Fig. 5. Estimated and experimental characteristics of SVD-1 stage at $\mathrm{Re}_{u}=1 \cdot 10^{7}$ in section $2-2$; Inlet pressure $\mathrm{P}=0.4 \mathrm{MPa}$, actuating medium - nitrogen.

Figures 6, 7 show the calculated and experimental characteristics of the SVD - 1 at an inlet pressure of 1 $\mathrm{MPa}$. Calculation at an inlet pressure of $1 \mathrm{MPa}$ was carried out taking into account the roughness; the equivalent sand roughness was set.

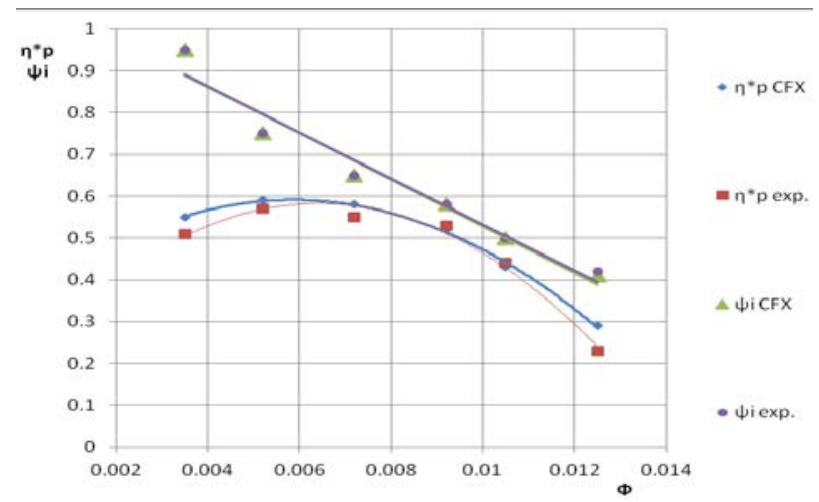

Fig. 6. A comparison of experimental characteristic of SVD-1 stage at $\operatorname{Re}_{u}=2,5 \cdot 10^{7}$ with estimated in section 2-2. Inlet pressure $\mathrm{P}=1 \mathrm{MPa}$, actuating medium - nitrogen.

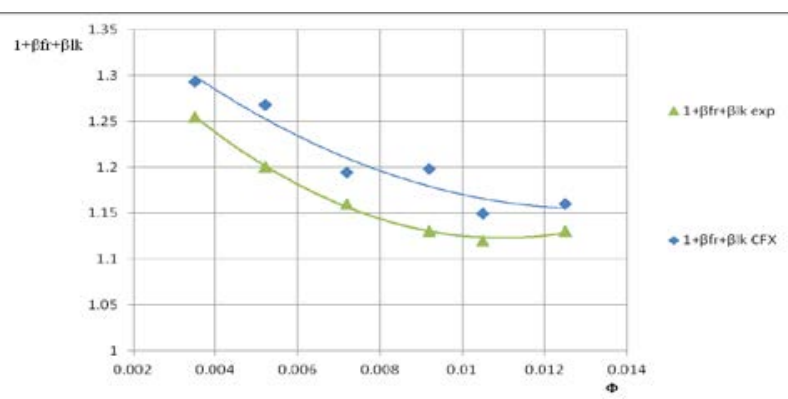

Fig. 7. Estimated and experimental characteristics of cumulative leak and disk friction loss factor $\left(1+\beta_{\mathrm{fr}}+\beta_{\mathrm{lk}}\right)$. Inlet pressure $\mathrm{P}=1 \mathrm{MPa}$, actuating medium - nitrogen .

Figures 8 show the characteristics of the model stage SVD -2 according to the results of a numerical study in comparison with the experimental results. The calculated input pressure is $0.1 \mathrm{MPa}$ of air ideal gas. The characteristics are built in section 2-2.

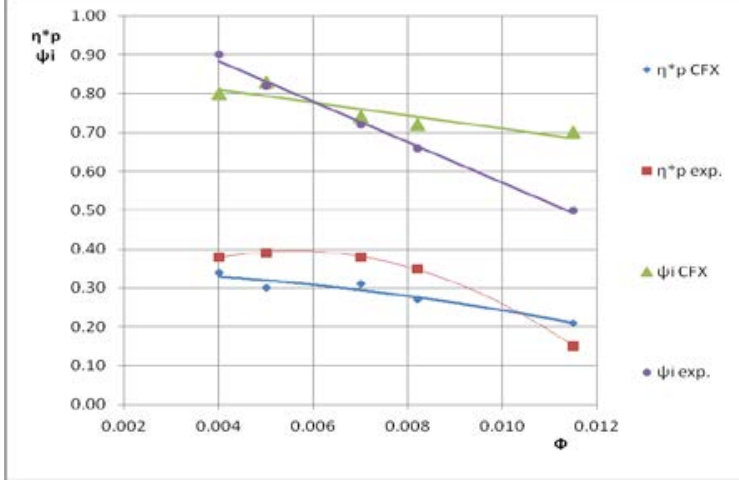

Fig. 8. Estimated and experimental characteristics of SVD-2 stage in section $2-2$.

Figure 9 show the characteristics of the model stage SVD -2 according to the results of a numerical study in comparison with the experimental results. Inlet pressure $\mathrm{P}=0.4 \mathrm{MPa}$, actuating medium - nitrogen.

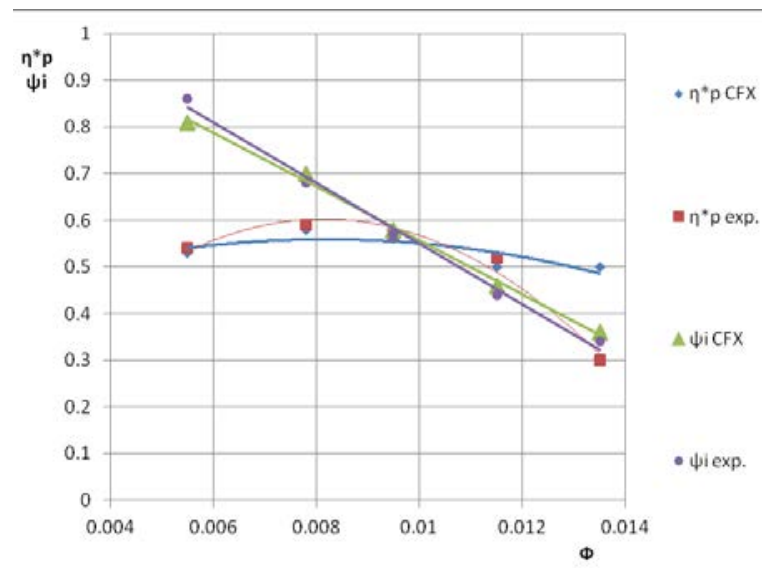

Fig. 9. Estimated and experimental characteristics of SVD-2 stage in section $2-2$. Inlet pressure $\mathrm{P}=0.4 \mathrm{MPa}$, actuating medium - nitrogen.

Figure 10 show the characteristics of the model stage SVD -2 according to the results of a numerical study in comparison with the experimental results. The calculated inlet pressure is $0.1 \mathrm{MPa}$ of air ideal gas. The characteristics are built in section 2-2.

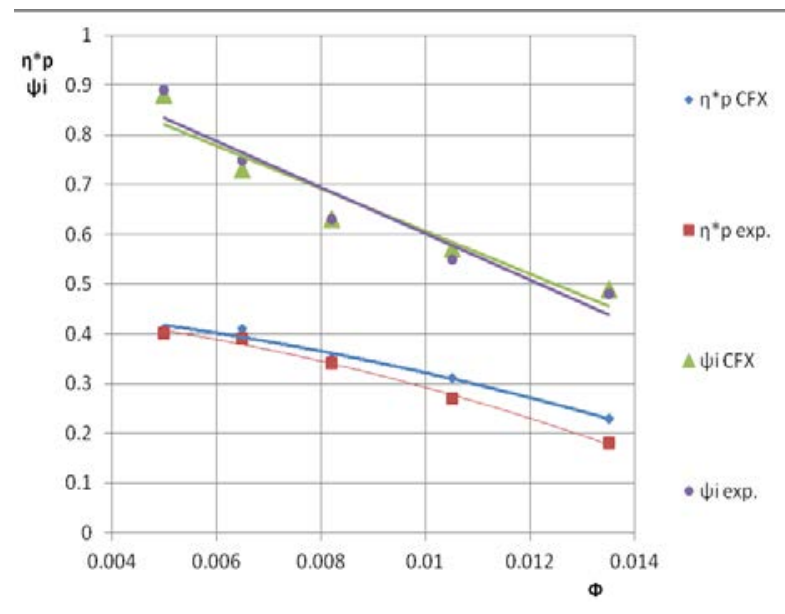

Fig. 10. Estimated and experimental characteristics of SVD-6 stage in section 2-2. Inlet pressure is $0.1 \mathrm{MPa}$ of air ideal gas. 
Figure 11 show the characteristics of the model stage SVD -6 according to the results of a numerical study in comparison with the experimental results. Inlet pressure $\mathrm{P}=2 \mathrm{MPa}$, actuating medium - nitrogen.

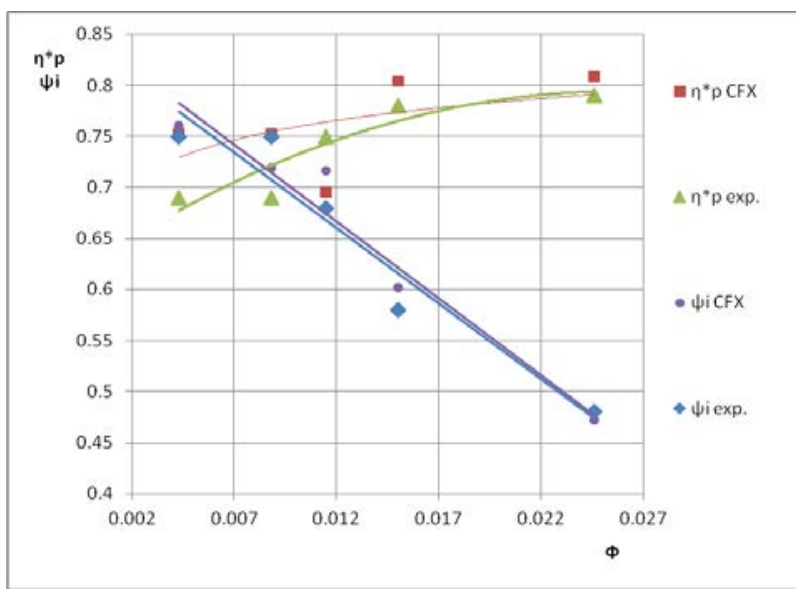

Fig. 11. Estimated and experimental characteristics of SVD-6 stage in section 2-2.. Inlet pressure $\mathrm{P}=2 \mathrm{MPa}$, actuating medium - nitrogen.

\section{Conclusions}

The study showed that in the viscid gas flow numerical simulation of low flow coefficient stages exist features which are related to an overestimation of the pressure characteristic for the flow rates greater than the nominal in atmospheric inlet pressure conditions. However, the opposite effect was obtained for the SVD-2 stage. The pressure curve and the efficiency curve will change when the domain of the vaneless diffuser is rotated with the walls braking to zero.

It is expected that under atmospheric input conditions, this approach will make it possible to bring the calculated characteristics closer to the experimental ones.

Also, during the study, it was found that the curve of the total loss coefficient for leaks and disk friction $(1+$ $\beta_{\mathrm{fr}}+\beta_{\mathrm{lk}}$ ) for SVD-1 under atmospheric input conditions is very close to the experimental curve. But the calculated characteristic increased by $5-7 \%$ for other input conditions. This is possibly explained by differences in the roughness of the surfaces of the model and the real object (the equivalent sand roughness was set for numerical investigation).

The size of the computational grid in seals minimally affects the results of a numerical study. The same results were obtained when the size of the first parietal cell was 0.001 and $0.01 \mathrm{~mm}$. Also, a temperature increase of up to $50^{\circ} \mathrm{C}$ in the labyrinth seals near the impeller walls was noted in the calculation. But it does not overestimate the temperature values in the section behind the impeller and does not cause an artificial increase in the internal head of the stage. This temperature grows in the stage seals can be caused by the energy supply from the rotating disk. While in the real impeller flow this is not possible. In general, the simulation results of the SVD stages characteristics are very similar with the real characteristics when using nitrogen with input conditions of $0.4 ; 1.0$ and $2.0 \mathrm{MPa}$.

\section{References}

1. Strizhak L.Ya. Thermogasdynamic Design Fundamentals of Centrifugal Compressors of High and Ultrahigh Pressure: dissertation of the Doctor of Engineering Sciences. Saint Petersburg: St.-Petersburg State Technical University; 1995. (In Russ.)

2. Sadovsky N.I. Increased Efficiency of Low Consumption Stages of High and Ultrahigh Centrifugal Compressors Based on the Knowledge of the Reynolds Number and Roughness Effect on Basic Processes: dissertation of the Candidate of Engineering Sciences. Saint Petersburg: St.-Petersburg State Technical University; 1994. (In Russ.)

3. Vlasov V.M. Development of a Designing Technique for Low Consumption Stages of High Pressure Centrifugal Compressors Based on a Real Gas Viscid Flow Model: dissertation of the Candidate of Engineering Sciences. Saint Petersburg: St.-Petersburg State Technical University; 1995. (In Russ.)

4. Izmailov R.A. Nonstationary Aerodynamic Processes in Centrifugal Compressors: dissertation of the Doctor of Engineering Sciences. Leningrad: M.I. Kalinin Leningrad Polytechnical Institute; 1989. (In Russ.)

5. Seleznev K.P., Galerkin Yu.B. Centrifugal Compressors. Leningrad: Mashinostroenie; 1982. (In Russ.)

6. Lebedev, A., Gileva, L., Danilishin, A., Sokolov, M.Surge protection system development in centrifugal compressor with an indicative method using numerical simulation of unsteady processes and analysis of pressure fluctuation signals. (2018) MATEC Web of Conferences, 245, paper № 09010.DOI: 10.1051/matecconf/201824509010

7. Ivanov, E.A., Zharkovsky, A.A., Borshchev, I.O., Svoboda, D.G. Technique for axial pump characteristics predicting in CFD package Openfoam. AIP Conference Proceedings v.2141,\#030063. (2019). DOI: 10.1063/1.5122113.

8. Zhurkin, N., Donskoj, A., Zharkovskij, A. Numeric modeling and estimating the performance characteristics of a pneumatic driven high pressure pump. (2018) MATEC Web of Conferences, 245, \# 09014. DOI: 10.1051/matecconf/201824509014.

9. V. V. Neverov, Y. V. Kozhukhov, A. M. Yablokov, A. A. Lebedev Optimization of a centrifugal compressor impeller using CFD: the choice of simulation model parameters 10th International Conference on Compressors and their Systems IOP Conf. Series: Materials Science and Engineering 232 (2017) 012037

10. Zharkovskii, A.A., Pospelov, A.Y. Use of 3D Methods for Flow Analysis, Prediction of Characteristics, and Optimization of the Shape of Settings of Hydraulic Turbines. (2015) Power Technology and Engineering, 49 (1), pp. 27-32.

11. Borm O, Kau H-P, Unsteady aerodynamics of a centrifugal compressor stage-validation of two different 
CFD solvers 2012 Proceedings of ASME Turbo Expo 2012, GT2012, Copenhagen, Denmark, June 11-15, GT2012-69636

12. Le Sausse P, Fabrie P, Arnou D, Clunet F 2013 CFD comparison with centrifugal compressor measurements on a wide operating range EPJ Web of Conferences, Vol. 45, p. 01059

13. Borm O, Balassa B, Kau H-P Comparison of different numerical approaches at the centrifugal compressor radiver 2011 ISABE Conference, International Society for Airbreathing Engines, Gothenburg, Sweden, $12^{\text {th }}-16^{\text {th }}$ September 2011 ISABE-2011-1242

14. M. Elfert, A. Weber, D. Wittrock, A. Peters, C. Voss, E. Nicke. Journal of turbomachinery 139(10) (2017).

15. T. Wang, C. Peng, J. Wu. Back swept angle performance analysis of centrifugal compressor MECHANIKA. (402-406) 20(4) (2014).

16. Pospelov, A.Y., Zharkovskii, A.A. Effect of the Parameters of a Computational Model on the Prediction of Hydraulic Turbine Characteristics. (2015) Power Technology and Engineering, 49 (3), pp. 159-164.

17. M. Elfert, A. Weber, D. Wittrock, A Peters, C.Voss, E. Nicke. Journal of turbomachinery 139(10), 101007 Doi:10.1116/1.4036357 (2017)

18. C. $\mathrm{Xu}$ and R. S. Amano. Meridional Considerations of the Centrifugal Compressor Development Hindawi Publishing Corporation International Journal of Rotating Machinery 2012 ID 518381,doi:10.1155/2012/518381(2012) 